[65] M. G. Ten Horst. Neth. Milk Dairy Journal, 1950, 4, 246.

[66] M. G. Ter Horst. Neth. Milk. Dairy Journal, 1947, 1, 137.

[67] A. Tiselius. Cité par numéro 37.

[68] VAN Den Bura. Cité par Mocquot, IXe Congrès International Ind. Agric., Rome, 1952, C. P. 20.

[69] L. L. VAN Slyke, A. W. Bosworth. Journal Biol. Chem., 1915, 20, no 2.

[70] C. H. Verdier. Nature (London), 1952, 170, 804.

[71] R. C. WARNER. Journal Amer. Chem. Soc., 1944, 66, 1.725.

[72] R. C. WARNer, E. Polis. Journal Am. Chem. Soc., 1945, 67, 529.

[73] E. G. Weir, A. B. Hasting. Journal Biol. Chem., 1936, 114, 397.

[74] D. W. Wooluex, a) Journal Am. Chem. Soc., 1945, 67, 1.734; b) $V I I I^{\mathrm{e}}$ Congrès de Chimie-biologique, Paris, 1948.

\title{
L'EMPLOI DES ULTRA-SONS POUR L'ASSAINISSEMENT DU LAIT
}

\section{par \\ le Professeur Corkado PACI}

On sait que le lait, même produit dans les meilleures conditions de milieu ambiant, renferme une riche flore microbienne $: 30.000$ à 200.000 germes par centimètre cube quand il est récolté proprement, 500.000 à 10.000 .000 quand la propreté et l'hygiène sont négligées. Il s'agit soi t de germes banaux, soit de germes utiles en fromagerie comme les ferments lactiques, ou industriellement nocifs comme les bacilles Coli et les bacilles butyriques, ou souvent même de germes spécifiques de maladies infectieuses transmissibles à l'homme : tuberculose bovine, brucellose, salmonellose, fièvre " $Q$ », etc., qui dans les cas les plus favorables, en raison des toxines produites par ces organismes, augmentént le degré déjà élevé d'instabilité du lait. Le problème de l'assainissement du lait contaminé à l'origine ou au cours du transport, en raison de ses conséquences sur l'alimentation humaine et sur les utilisations industreilles du lait lui-même, représente donc un grand problème économique, hygiénique et social, qui n'a pas encore reçu de solution satisfaisante. L'ébullition (parfois incomplète) à laquelle on a coutume de soumettre le lait avant la consommation, chez l'utilisateur, constitue dans la majeure partie des cas un moyen optimum d'assainissement, mais cela au prix de transformations profondes du produit qui portent un grave préjudice aux caractères organoleptiques et aux très précieuses propriétés biochimiques. La pasteurisation résout en grande partie ce problème, mais au prix d'une dépense excessive et en communiquant au lait pasteurisé un goût caractéristique de (" cuit " qui ne plaît pas au consommateur. D'autre part, la pasteurisation n'élimine pas les produits de méta-

(1) La Clinica Veterinaria, septembre 1952. 
bolisme des bactéries qui se sont développées avant le traitement thermique. Pour cette raison, on étudie actuellement des systèmes d'assainissement du lait aptes à conserver à ce produit extrêmement instable son arome et sa saveur originales, et à épargner le plus complètement possible ses propriétés biochimiques. On connaît par exemple, au moins par leur principe théorique, la méthode de la pasteurisation sous vide; le traitement à l'eau oxygénée selon la méthode Nai-Giolitti ; la congélation ultra-rapide à très basse température selon la méthode Antoniani; le traitement aux rayons ultra-violets, auquel on doit ajouter celui des ultra-sons, objet de recherches pașsionnées aux Etats-Unis, au Japon, en Allemagne et dans notre pays lui-même.

C'est vers le début du siècle que l'attention des physiciens a été attirée par les ultra-sons, vibrations longitudinales élastiques semblables à celles du son, mais non perceptibles en raison de leur haute fréquence d'oscillation. La sensibilité de l'oreille humaine est. généralement comprise entre 16 et 16.000 Hertz. L'Hertz équivaut à une oscillation par seconde. Des oscillations ou fréquences inférieures à 16 Hertz constituent la caractéristique des infra-sons, dont les manifestations les plus connues sont les ondes sismiques : tremblements de terre, raz de marée, etc. Les oscillations ou fréquences supérieures à 16.000 Hertz caractérisent les ultra-sons, qu'il est possible de capter au moyen d'appareils enregistreurs spéciaux, et qui sont seulement audibles par quelques espèces d'animaux.

Le public profane connaît aussi le phénomène surprenant du sifflement ultra-sonique émis par une sirène portative, sifflement inaudible par l'homme, mais distinctement perceptible dans toutes ses modulations par le chien policier. Les grillons, les papillons nocturnes, les pipistrelles, émettent et reçoivent des ultra-sons de très haute fréquence. Le cri émis par les pipistrelles est constitué par une succession de signaux à haute fréquence - environ 50.000 Hertz - d'une durée d'un deux centièmes de seconde, émis périodiquement à des intervalles de quelques dixièmes de seconde. Les pipistrelles se servent des échos réfléchis de ces signaux pour régler leur vol, afin d'éviter les obstacles, de la même façon que l'avion guidé par le radar. Les ultra-sons sont de plus en plus utilisés en thérapeutique et en biologie, au moyen d'appareils générateurs relativement simples et peu encombrants qui utilisent l'effet piézo-électrique découvert par CuBIE, effet par lequel l'énergie électrique se transforme en énergie mécanique avec production de chaleur, par l'excitation d'un mince cristal de quartz. Ainsi, une lame de quartz, placée dans le voisinage d'un champ électrique alternatif convenablement orienté, transforme les 
décharges électriques à très haute fréquence qu'elle reçoit en vibrations mécaniques d'égale fréquence. Ces vibrations peuvent être transmises à un liquide ou à un tissu vivant par contact direct, au moyen de dispositifs spéeiaux transmetteurs, dont l'intensité de propagation est conditionnée par la puissance acoustique de lappareil générateur, puissance qui s'exprime en Watts par centimètre carré. Pour s'exprimer le plus simplement, on peut dire avec Carbonini que les ondes ultra-soniques réalisent un véritable et parfait moyen de transport de l'énergie mécanique, qui est pour sa plus grande partie absorbée par le milieu support, et transformée en autant de manifestations de nature mécanique, thermique, chimique, de la plus haute valeur en raison précisément de leur pouvoir d'action biologique ou thérapeutique. Le mécanisme des actions des ultra-sons, dans les liquides et dans les tissus organiques, peut être ramené schématiquement à des actions catalytiques, à des actions oxydantes, polarisantes ou dépolarisantes, ou à des actions de dissociation de composés chimiques complexes, avec formation d'une nouvelle substance intermédiaire, et avec détermination dans le milieu support de brusques passages par divers stades de constitution physiques, jusqu'à parvenir à la coagulation et à la précipitation des protéines, et aux plus violentes actions perturbatrices et destructrices des cellules et des tissus. L'application des ultrasons se rencontre déjà sur une grande échelle en thérapeutique, pour le traitement de tumeurs malignes cutanées, d'eczémas, d'ulcères, etc., et dans le domaine bactériologique et de l'hygiène alimentaire, ils sont utilisés en raison de leur puissante action bactéricide. La destruction des bactéries en suspension dans un liquide est principalement fonetion de la fréquence des vibrations et de la durée du traitement. En général, on utilise pour cette destination des fréquences de 175 à $1.500 \mathrm{~K}$. Hertz $(1 \mathrm{~K}$. Hertz équivaut à $1.000 \mathrm{Hertz}$ ), et des appareils générateurs d'une puissance de 45 à 60 Watts acoustiques, avec une intensité de propagation de l'ordre de 2 à 3 Watts par centimètre carré. La durée du traitement peut varier de deux à vingt minutes. Bien que les résultats soient extraordinairement variables selon la limpidité et la densité du milieu, ainsi que d'autres circonstances non encore parfaitement éclaircies, il est certain qu'à partir de 0,4 Watts par centimètre carré et avec des fréquences de 170 à $1.000 \mathrm{Hertz}$, il est possible de détruire dans une large mesure des colonies microbiennes et des virus.

Dans le lait, le premier essai de stérilisation à l'aide des ultrasons a été accompli par L. A. Chamber et N. Gaines en 1932, lesquels, avec des moyens de laboratoire rudimentaires, ont réussi à détruire $80 \%$ de la flore bactérienne du lait ordinaire. Successi-

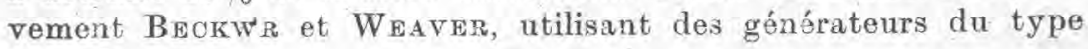


piézo-jec'rione à forte puissance, obtinrent la destruction presque totale de la flore bactérienne sans toutefois parvenir à la stérilisation, et avec le grave inconvénient d'un fort développement de chaleur. Plus tard, en 1946, StempF GReen démontra l'action limitatrice de la densité du milieu sur la propagation des ultrasons, et la fonction protectrice, pour les bactéries, exercée par les protéines. Enfin dernièrement, dans l'Illinois, Stanley, Whitney et Russel déclarèrent, avoir obtenu des résultats pratiquement positifs dans le traitement du lait, et en Amérique, des installationspilote fonctionnent déjà pour la pasteurisation et l'homogénéisation du lait. Mais il y a encore de nombreuses difficultés à surmonter avant que ce puissant moyen d'assainissement et de conservation du lait entre dans la pratique industrielle. Le lait, à la différence des autres liquides alimentaires, présente des difficultés particulières de traitement en raison de sa nature colloïdale qui réduit l'action biochimique des ultra-sons. Car la présence des protéines complexes en suspension ou répandues dans le plasma du lait, constitue une protection physique efficace pour les microorganismes. Il est cependant certain que l'action des ultra-sons est positive à $100 \%$ pour le groupe Coli, les bacilles typhiques et de la diphtérie ; partiellement positive pour les "coques", les streptocoques et les Brucella. L'action est nulle pour le bacille du charbon. Le bacille de la tuberculose est détruit à raison d'une proportion élevée, mais pas encore totalement. Les vitamines ne sont aucunement atteintes, pas même les plus instables comme la B1, la B6, et la C. Par contre, les enzymes sont détruites complètement, comme cela se passe d'ailleurs avec la pasteurisation, et à plus forte raison avec l'ébullition chez le consommateur. Comme effet utile, on doit relever en premier lieu le fractionnement des globules gras, par lequel, dans le lait traité par les ultra-sons, on réaliserait outre l'effet bactéricide, celui de l'homogénéisation. Et ce dernier résultat n'est pas le moins important. Comme on sait que dans le lait homogénéisé à la suite de traitements mécaniques de choc (auxquels on peut d'ailleurs comparer l'action des ultra-sons), les globules gras se réduisent de volume, offrant une plus grande surface d'attaque aux ferments lipolytiques, pour lesquels le lait homogénéisé est plus digestible. Witric remarque en outre que la réduction dimensionnelle des globules gras et l'augmentation de la surfáce d'attaque de la matière grasse ne sont pas le seul résultat de l'homogénéisation du lait, bien qu'en étant la conséquence la plus clairement visible. Il faut aussi constater une profonde modification de l'état physique de la caséine et de son complexe colloïdal sous l'influence du phénomène d'adsorption, et notamment de la formation de nouvelles membranes autour des globules gras résultant de cette subdivision. Pour cette 
raison, la caséine, parvenue dans l'estomac, est précipitée sous l'action de l'acide chlorhydrique contenu dans le sue gastrique en coagulums beaucoup plus fins et moins consistants que ceux que présentait le lait à l'origine, parce qu'ils sont plus facilement et plus profondément attaqués par les ferments protéolytiques. Ces phénomènes revêtent une importance particulière dans l'allaitement artificiel des nourrissons, qui accusent souvent une sensibilité anormale pour le lait de vache.

Wiтtig soutient à ce propos que de nombreux désordres gastriques provoqués par l'ingestion de lait et une grande partie des cas d'intolérance vis-à-vis de cet aliment, sont imputables à l'incapacité d'une partie des ferments lipolytiques de dissocier complètement la matière grasse dans l'intestin, et à une coagulation trop grossière de la caséine dans l'estomac. En outre, le lait homogénéisé ne donne pas lieu (ou presque pas) à la formation de pellieule à la surface du récipient pendant l'ébullition, et il ne peut être écrémé en raison de l'impossibilité pour une partie des globules gras de remonter dans le plasma du lait. Par contre, il peut se produire que dans le lait homogénéisé, soit par les traitements " de choe ", soit par l'effet des ultra-sons, on constate un commencement d'hydrolyse de la matière grasse, avec une modification correspondante de la saveur, phénomènes qui pourront certainement être empêchés par une technique plus perfectionnée. En attendant, dans l'intention de surmonter les dernières difficultés pour la mise au point de ce moyen d'assainissement du lait, qui promet les applications les plus intéressantes du point de vue économique et social dans le domaine de l'hygiène alimentaire, des recherches fébriles sont partout en cours, sur lesquelles on observe naturellement une réserve bien explicable. Dans notre pays également, malgré les conditions de pauvreté auxquelles est réduire la recherche scientifique, le problème de l'assainissement du lait au moyen de l'emploi des ultra-sons est l'objet de recherches très détaillées. A l'Institut de Mierobiologie Agricole de l'Université de Milan, dirigé par le Professeur Carlo ARnadd, on a pu établir que la fréquence de $1.000 \mathrm{~K}$. Hertz est suffisante pour parvenir à une action intense microbicide, même avec des appareils d'une puissance limitée à 70 Watts et avec un développement de chaleur non supérieur à $10^{\circ} \mathrm{C}$. Dans ces conditions, ARNAud et ses Collaborateurs, adoptant une méthode spéciale au moyen de laquelle l'émis. sion ultra-sonique est distribuée de façon intermittente, ont obtenu un effet microbicide de l'ordre de $80 \%$, avec des traitements d'une durée de quinze minutes, une puissance acoustique de 55 Watts et un développement de chaleur non supérieur à $8^{\circ} \mathrm{C}$. Ces résultats, parmi lesquels le plus important pour la conservation des carac- 
tères organoleptiques du lait réside dans l'abaíssement de la température, sont déjà une base d'une importance décisive pour des investigations ultérieures plus approfondies, qui seront entreprises au cours des prochains mois, avec des appareils de plus grande puissance et conçus de façon à obtenir le traitement du lait dans le liquide circulant. Le progrès très rapide réalisé dans la technique des ultra-sons au cours de ces deux dernières années permet de prévoir que le moment n'est pas loin où l'on pourra employer pour le lait et sur une grande échelle ce moyen physique énergiquement antimicrobien, qui possède entre autres le privilège d'agir à températures moyennes, sans causer aucune modification dans le " patrimoine" biologique du lait, et en grande partie dans sa constitution colloïdale, à part l'action bénéfique exercée sur la matière grasse.

\title{
TRAVAUX ET RECHERCHES SUR LE LAIT ET LES PRODUITS LAITIERS
}

\author{
(Fin.)
}

\section{MÉTHOdES ANALYTIQUES DU LAIT} (Section bactériologie) $(x)$

\section{Raisons de la création de la sous-commission de contrôle bactériologique du lait}

Alors que le contrôle des aérobies est depuis longtemps réalisé, on n'avait jamais pensé à organiser celui des anaérobies des laits crus, pasteurisés et des fromages. On s'est aperȩu des dangers de cette lacune à l'Armée d'Extrême-Orient, où une proportion très élevée de boîtes de fromage fondu ont été avariées par les anaérobies et en particulier par Pl. tetani.

Un comité de bactériologistes et d'industriels a été réuni pour réparer cette lacune.

Les travaux se sont déroulés parallèlement au Service des Anaérobies de l'Institut Pasteur et dans les laboratoires industriels.

\section{Recherches expérimentales et industrielles}

A. - Les faits cités plus haut d'une proportion très élevée de boîtes avariées de fromage fondu à l'Armée d'Orient montre que les anaérobies putréfiants et destructeurs peuvent s'y développer tardivement et entraîner la perte de denrées alimentaires. Il était logique de se demander en plus si le lait et ses dérivés pouvaient transmettre les maladies anaérobies.

(1) Rapport présenté par M. Prévot. 